\title{
A convenient and efficient conversion of 2-carboxyheteroarenes into $N$-(2-thienyl- and 2-selenophenyl)-1-methylpyrrole-2-carboxamides
}

\author{
Filippo Danielli and Paolo Zanirato*
}

\author{
Dipartimento di Chimica Organica, 'A. Mangini', Università di Bologna, viale Risorgimento 4, \\ 40136 Bologna, Italy \\ E-mail: zanirato@ms.fci.unibo.it
}

(received 26 Dec 99; accepted 13 Feb 00; published on the web 21 Feb 00)

DOI: http://dx.doi.org/10.3998/ark.5550190.0001.109

\begin{abstract}
Nitrogen-linked five-membered heteroaryls are obtained by conversion of 2-carboxyheteroarenes into $\mathrm{N}$-(2-heteroaryl)-1-methylpyrrole-2-carboxamides. The procedure is based on a simple thermal rearrangement of thiophene or selenophene carbonyl azides in neat 1methylpyrrole at $90{ }^{\circ} \mathrm{C}$.
\end{abstract}

Keywords: Thermal rearrangement, carbonyl azides, thiophene

\section{Introduction}

Pseudohalides, as azide and isocyanate, are important functional groups in synthetic organic chemistry and have been widely used as regio- and stereo-controlled precursors of the amino function. ${ }^{1}$ The azido transfer reaction of lithiated five-membered 2- and 3-heteroaryls with tosyl azide provides a convenient entry to the otherwise problematic nitrogen-linked heteroaryls containing one heteroatom ${ }^{2}$ but, unfortunately, there are drawbacks. ${ }^{3}$ The 2-heteroaryl azides show a general tendency to suffer from a low-temperature ring cleavage fragmentation and are often only formed in low yield. ${ }^{4}$ The conversion of certain aryl isocyanates into amines has been investigated $^{1 \mathrm{c}}$ and those derived from five-membered heteroaryls remain virtually unexplored, although their preparation is readily feasible from available carbonyl azido precursors. ${ }^{5}$

The literature contains a number of similar procedures for the conversion of heteroaryl isocyanates into amines, ${ }^{5}$ and these have been classified according to their stability. Most such procedures deal with the conversion of the initial isocyanate into amides under protic or Lewis acid conditions, or by the addition of organometallic reagents. ${ }^{6}$

In recent years thiophenecarbonyl azides, normally available from the corresponding carboxylic acid via the acyl chloride and trimethylsilyl azide, have been valuable materials in nitrogenlinked thiophene synthesis. ${ }^{7}$ 
As a continuation of our research on the synthesis of nitrogen-linked five-membered heteroaryls we now report an efficient conversion of 2-carboxyheteroarenes into $N$-(2-heteroaryl)-1methylpyrrole-2-carboxamides. The procedure is based on a simple thermal rearrangement of heteroaryl carbonyl azides 1a-6a in neat 1-methylpyrrole.

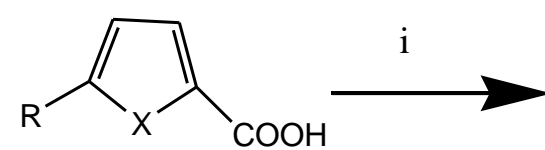

$\mathrm{i}=\mathrm{ClCO}_{2} \mathrm{Me}, \mathrm{Me}_{3} \mathrm{SiN}_{3}$;

ii = 1-methylpyrrole, $90^{\circ} \mathrm{C}$;<smiles>[R]c1[X]c(C(N)=O)cc1</smiles>

1a-6a

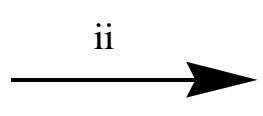

$1: \mathrm{R}=\mathrm{H}, \mathrm{X}=\mathrm{S}$

$2: \mathrm{R}=\mathrm{H}, \mathrm{X}=\mathrm{Se}$

$3: \mathrm{R}=\mathrm{H}, \mathrm{X}=\mathrm{O}$

$4: \mathrm{R}=\mathrm{Me}, \mathrm{X}=\mathrm{S}$

$5: \mathrm{R}=\mathrm{SiMe} 3, \mathrm{X}=\mathrm{S}$

6:R=SiMe3, $\mathrm{X}=\mathrm{Se}$

A number of synthetic analogues of the potent antitumoral distamycin and netropsin have been found to have excellent cytotoxicity and anticancer activity. The 1-methylpyrrole-2carboxamides, formed by amino-heteroaryl derivatives, are of special interest as a potential nitrogen mustard of the distamycin type ${ }^{8}$ and the heteroaryl amide linkage might be considered to play a synergistic role on bioactivity. ${ }^{9}$

\section{Results and Discussion}

The starting carbonyl azides 1a-6a were prepared in high yields (90-72\%) from readily available carboxylic acids by the procedure developed by Weinstock, ${ }^{10}$ partially modified by using the less hazardous treatment of mixed carboxylic-carbonic anhydrides with trimethylsilyl azide instead of acyl chlorides and sodium azide

Significantly 1-methyl- $N$-(2-thiophene)-(1), 1-methyl- $N$-(2-selenophene)-(2) and 1-methyl- $N$-(2furano)-(3) -1H-pyrrole-2-carboxamide are obtained in good yields by mild thermal rearrangement (at $90{ }^{\circ} \mathrm{C}$ ) of the appropriate carbonyl azides 1a, 2a and 3a in 1-methylpyrrole

Table 1. Yields, physical features and IR, MS and $\delta_{H}$ NMR spectral data of carboxamides 1-6

\begin{tabular}{|c|c|c|c|c|c|c|c|c|c|c|c|c|c|}
\hline & & & $\operatorname{IR}(\mathrm{f}$ & & MS & $\begin{array}{l}\delta(\mathrm{ppn} \\
\mathrm{CDC}\end{array}$ & $200 \mathrm{M}$ & & & & & & \\
\hline $\begin{array}{l}\text { compd } \\
\text {. }\end{array}$ & $\begin{array}{l}\text { Yield } \\
(\%)\end{array}$ & $\mathrm{mp} /{ }^{\circ} \mathrm{C}$ & $\mathrm{NH}$ & $\mathrm{CO}$ & $\begin{array}{l}\mathrm{m} / \mathrm{z} \\
(\mathrm{M}+)\end{array}$ & H-3 & $\mathrm{H}-4$ & H-5 & H-3' & H-4' & H-5' & $\mathrm{NH}$ & $\mathrm{Me}$ \\
\hline 1 & 83 & $170-72$ & $\begin{array}{l}330 \\
0\end{array}$ & $\begin{array}{l}163 \\
5\end{array}$ & 206 & 6.70 & 6.88 & $6.87^{\mathrm{a}}$ & 6.75 & 6.15 & $\begin{array}{l}6.81 \\
\text { e }\end{array}$ & 8.27 & 4.00 \\
\hline 2 & 70 & $175-77$ & $\begin{array}{l}329 \\
5\end{array}$ & $\begin{array}{l}163 \\
0\end{array}$ & 254 & 6.78 & 7.12 & $7.54^{\mathrm{b}}$ & 6.75 & 6.16 & $\begin{array}{l}6.81 \\
\mathrm{e}\end{array}$ & 8.58 & 4.00 \\
\hline
\end{tabular}




\begin{tabular}{|c|c|c|c|c|c|c|c|c|c|c|c|c|c|}
\hline 3 & 74 & resinous & $\begin{array}{l}330 \\
0\end{array}$ & $\begin{array}{l}166 \\
0\end{array}$ & 190 & 6.33 & 6.39 & $7.08^{\mathrm{c}}$ & 6.70 & 6.13 & $\begin{array}{l}6.79 \\
\text { e }\end{array}$ & 7.96 & 3.97 \\
\hline 4 & 68 & resinous & $\begin{array}{l}323 \\
0\end{array}$ & $\begin{array}{l}163 \\
0\end{array}$ & 220 & 7.64 & $6.78^{\mathrm{d}}$ & - & 6.75 & 6.11 & $\begin{array}{l}6.70 \\
\text { e }\end{array}$ & 8.33 & $\begin{array}{l}4.00 \\
/ 2.52^{\mathrm{f}}\end{array}$ \\
\hline 5 & 81 & res. oil & $\begin{array}{l}329 \\
5\end{array}$ & $\begin{array}{l}163 \\
0\end{array}$ & 278 & 6.75 & $6.98^{\mathrm{d}}$ & - & 6.74 & 6.13 & $\begin{array}{l}6.77 \\
\mathrm{e}\end{array}$ & 8.38 & $\begin{array}{l}4.01 \\
/ 0.30^{\mathrm{g}}\end{array}$ \\
\hline 6 & 78 & res. oil & $\begin{array}{l}330 \\
0\end{array}$ & $\begin{array}{l}163 \\
5\end{array}$ & 326 & 6.90 & $7.31^{\mathrm{d}}$ & - & 6.64 & 6.17 & $\begin{array}{l}6.81 \\
\mathrm{e}\end{array}$ & 8.78 & $\begin{array}{l}4.00 \\
/ 0.32^{\mathrm{g}}\end{array}$ \\
\hline
\end{tabular}

$J$ are in $\mathrm{Hz} ;{ }^{a} J_{\mathrm{Th}} 5.5,3.7,1.5 ;{ }^{b} J_{\mathrm{Se}} 6.1,4.0,1.3 ;{ }^{c} J_{\mathrm{Fu}} 3.3,2.0,1.05 ;{ }^{d} J_{34} 3.85 ;{ }^{e} J_{\mathrm{Py}} 4.05,2.6,1.7 ;{ }^{f} \mathrm{Me}, J_{\mathrm{Me}-4} 1.0 ;{ }^{g}$ $\mathrm{SiMe}_{3}$

This study was likewise extended to para-like substituted thiophene carbonyl azides $4 \mathbf{a}$ and 5a $(\mathrm{R}=\mathrm{Me}, \mathrm{SiMe} 3 ; \mathrm{X}=\mathrm{S})$, and selenophene $\mathbf{6 a}(\mathrm{R}=\mathrm{SiMe} 3 ; \mathrm{X}=\mathrm{Se})$ (Scheme 1). The reactions of 1a-6a were followed by GC-MS analysis. This analysis of the thermolysis mixtures supports the evidence that the rearrangement results almost exclusively in the formation of the corresponding methyl 1H-pyrrole-2-carboxamides 1-6 ( $/ 2 / z$ 206, 254, 190, 220, 278 and 326, respectively).

Products 1-6 were purified by chromatography and the precise structures were confirmed by: ${ }^{1} \mathrm{H}$ , ${ }^{13} \mathrm{C}-\mathrm{NMR}$, IR and high-resolution mass spectra (Table 1). The mass spectra (E.I $70 \mathrm{eV}$ ) exhibit appropriate molecular ions and a base peak corresponding to the favourable cleavage of the $\mathrm{C}-\mathrm{N}$ amide bond to give the 1-methylpyrrole acyl cation $(\mathrm{m} / \mathrm{z}, 108)$ as the primary fragmentation product.

Moreover, the thermal reaction of 1a also takes place in neat pyrrole affording a high yield of (2thiophene)-1H-pyrrole-2-carboxamide, whose structural assignment was made on the basis of ${ }^{1} \mathrm{H}$ NMR spectroscopy as follows: $\delta_{\mathrm{H}}\left(200 \mathrm{MHz}, \mathrm{CDCl}_{3} / \mathrm{J} / \mathrm{Hz}\right) 7.15(1 \mathrm{H}, \mathrm{m}), 7.10(1 \mathrm{H}, \mathrm{m}), 7.02$ $(1 \mathrm{H}, \mathrm{q}, J 5.4$ and 1.6), $6.97(1 \mathrm{H}, \mathrm{q}, J 5.4$ and 3.7), $6.92(1 \mathrm{H}, \mathrm{q}, J 3.7$ and 1.6) and $6.29(1 \mathrm{H}, \mathrm{m})$. The pyrrole ring protons were confirmed by $\mathrm{D}_{2} \mathrm{O}$ exchange, giving a typical $\mathrm{ABX}$ system, $J$ being $1.45,2.50$ and $3.75 \mathrm{~Hz}$.

There is only one definite example of thermal attack of thiophenecarbonyl azide onto an adjacent pyrrole substituent. This is represented by intramolecular cyclization at $130{ }^{\circ} \mathrm{C}$ of $2-(1 \mathrm{H}$-pyrrol1-yl)-3-thiophenecarbonyl azide to pyrrolo[1,2-a]thieno[3,2-e]pyrazin-5-(4H)-one. ${ }^{11}$ Consequently, our intermolecular thermal fragmentation of heteroaryl carbonyl azides most likely involves a mechanism that requires further investigation. Photochemical Curtius rearrangements are often characterised by the final presence of nitrene trapping by-products; however thermolysis is a concerted process, faster than nitrene formation. ${ }^{12}$ On thermolysis of carbonyl azides 1a-6a in 1-methylpyrrole, the resulting pyrrole-2-carboxamides are presumed to involve the intermediacy of the corresponding isocyanate. In this case, the successful outcome of such thermal reactions is ascribable to the pronounced acidic property of the pyrrole 2-proton. ${ }^{13}$ In conclusion, in the present report we have shown that the thermal rearrangement of chosen heteroarylcarbonyl azides in neat 1-methylpyrrole (or pyrrole) can be usefully employed in the preparation of a series of potentially bioactive pyrrole- $N$-(2-heteroaryl)-2-carboxamides. 


\section{Experimental Section}

General Procedures. All reactions were carried out under nitrogen atmosphere. The ${ }^{1} \mathrm{H}-$ and ${ }^{13} \mathrm{C}-\mathrm{NMR}$ spectra were recorded at 200 and $50 \mathrm{MHz}$. The chemical shifts are reported in ppm $(\delta)$ relative to TMS in $\mathrm{CDCl}_{3}$ and $J$ values are given in Hz. The IR spectra were recorded in $\mathrm{cm}^{-1}$ on a Perkin Elmer 257 and mass spectra were recorded on a VG Analytical 7070E instrument. Carboxylic acids were prepared according to the literature. ${ }^{14}$

1-Methyl- $N$-(2-thiophene)-1H-pyrrole-2-carboxamide (1). Typical procedure. A solution of 2-thiophenecarbonyl azide $1 \mathrm{a}(5 \mathrm{mmol})$ in $4 \mathrm{~mL}$ of neat 1 -methylpyrrole was allowed to react in a sealed tube at $90{ }^{\circ} \mathrm{C}$ and in the dark for ca. $1 \mathrm{~h}$. After careful removal of the excess 1methylpyrrole under vacuum, the residue was purified by chromatography $\left(\mathrm{Al}_{2} \mathrm{O}_{3}\right)$ using dry petroleum ether (bp. 40-60 ${ }^{\circ} \mathrm{C}$ )/diethyl ether (1:1) as eluant: ${ }^{13} \mathrm{C}$ NMR: 37.4(q, $J$ 139.0), 108.1(d, $J$ 173.8), 111.9(d, $J$ 167.0), 112.7(s), 113.0(d, $J$ 168.6), 118.0(d, $J$ 186.5), 124.3(d, $J$ 168.3), 129.6(d, J 184.5), 139.5(s), 158.4(s). $\mathrm{m} / \mathrm{z}, 206\left(\mathrm{M}^{+}, 26.0 \%\right), 108(\mathrm{M}-98,100), 80(7.4), 53(17.1)$, 39(16.2) (Found: $\mathrm{M}^{+}, 206.0514, \mathrm{C}_{10} \mathrm{H}_{10} \mathrm{~N}_{2} \mathrm{OS}$ requires $\left.\mathrm{M}, 206.0514\right)$.

1-Methyl- $\boldsymbol{N}$-(2-selenophene)-1H-pyrrole-2-carboxamide (2). ${ }^{13} \mathrm{C}$ NMR: $37.4(\mathrm{q}, J$ 140.5), 108.2(d, $J$ 173.9), 111.8(d, $J$ 163.3), 112.7(s), 112.9(d, $J$ 168.5), 123.2(d, $J$ 187.0), 126.3(d, $J$ 163.1), 129.7(d, J 189.5), 141.7(s), 158.1(s). m/z, 254( $\left.\mathrm{M}^{+}, 13.6 \%\right), 108(\mathrm{M}-146,100), 80(6.5)$, 53(17.6), 39(12.3) ) (Found: $\mathrm{M}^{+}, 253.9958, \mathrm{C}_{10} \mathrm{H}_{10} \mathrm{~N}_{2}$ OSe requires M, 253.9958).

1-Methyl- $\boldsymbol{N}$-(2-furano)-1H-pyrrole-2-carboxamide (3). ${ }^{13} \mathrm{C}$ NMR: 37.3(q, $J$ 140.0), 95.6(d, $J$ 182.5), 108.1(d, $J$ 174.0), 112.0(d, $J$ 175.0), 113.2(d, $J$ 168.5), 113.5(s), 129.6(d, $J$ 184.5), 135.9(d, J 205.1), 145.8(s), 158.2(s). m/z, 190(M+1 17.5\%), 108(M-82, 100), 80(7.0), 53(13.6), 39(11.2) ) (Found: $\mathrm{M}^{+}, 190.0741, \mathrm{C}_{10} \mathrm{H}_{10} \mathrm{~N}_{2} \mathrm{O}_{2}$ requires $\mathrm{M}, 190.0742$ ).

1-Methyl- $\boldsymbol{N}$-(5-methyl-2-thiophene)-1H-pyrrole-2-carboxamide $\quad(4) .{ }^{13} \mathrm{C} \quad \mathrm{NMR}: \quad 15.2(\mathrm{q}$, 128.9), 37.3(q, $J$ 140.4), 108.0(d, $J$ 173.5), 112.1(d, $J$ 163.6), 112.8(d, $J$ 168.8), 114.0(s), 121.9(d, $J$ 165.5), 129.3(d, $J$ 184.2), 134.5(s), 151.4(s), 158.4(s). m/z, 220(M $\left.\mathrm{M}^{+}, 26.6 \%\right), 108(\mathrm{M}-$ 112, 100), 80(7.8), 53(18.7), 39(14.6) (Found: $\mathrm{M}^{+}, 220.0670, \mathrm{C}_{11} \mathrm{H}_{12} \mathrm{~N}_{2} \mathrm{OS}$ requires $\mathrm{M}$, 220.0670).

1-Methyl- $\boldsymbol{N}$-(5-trimethylsilyl-2-thiophene)-1H-pyrrole-2-carboxamide (5). ${ }^{13} \mathrm{C}$ NMR: $0.2(\mathrm{q}$, 119.7), 37.3(q, $J$ 140.3), 108.1(d, $J$ 173.8), 111.9(s), 113.1(d, $J$ 168.8), 113.5(d, $J$ 165.3), 129.6(d, $J$ 184.2), 131.5(d, $J$ 165.9), 144.4(s), 158.4(s). $m / z, 278\left(\mathrm{M}^{+}, 21.4 \%\right), 108(\mathrm{M}-170,100)$, 73(12.6), 57(6.5), 53(10.7), 39(9.0) (Found: $\mathrm{M}^{+}$, 278.0909, $\mathrm{C}_{13} \mathrm{H}_{18} \mathrm{~N}_{2} \mathrm{OSSi}$ requires M, 278.0909).

1-Methyl- $\boldsymbol{N}$-(5-trimethylsilyl-2-selenophene)-1H-pyrrole-2-carboxamide $\quad(6) . \quad{ }^{13} \mathrm{C} \quad \mathrm{NMR}$ : 0.7(q, 119.9), 37.4(q, J 140.3), 108.3(d, J 173.7), 111.9(s), 113.0(d, J 168.6), 113.9(d, $J$ 162.1), 129.8(d, $J$ 187.5), 133.5, (d, $J$ 165.9), 146.3(s), 158.1(s). $\mathrm{m} / \mathrm{z}, 326\left(\mathrm{M}^{+}, 21.4 \%\right), 108(\mathrm{M}-218,100)$, 73(12.6), 53(10.7), 39(9.0) (Found: $\mathrm{M}^{+}, 326.0355, \mathrm{C}_{13} \mathrm{H}_{18} \mathrm{~N}_{2} \mathrm{OSeSi}$ requires $\mathrm{M}, 326.0354$ ). 


\section{Acknowledgements}

The work was carried out as part of the 'Progetto di Finanzamento Triennale, Ateneo di Bologna'. Financial support from the Ministero dell'Università e della Ricerca Scientifica (MURST) is also acknowledged. P.Z. thanks Emeritus Professor Salo Gronowitz, whose support makes this work possible.

\section{References}

1. (a) Sheradsky, T. The Chemistry of the Azido Group, Patai, S., Ed.; Interscience: London, 1971, p 331. (b) Scriven, E. F. V.; Turnbull, K. Chem, Rev. 1988, 88, 297. (c) Chemistry and Technology of Isocyanates, Ulrich, H., Ed., Wiley: Chichester, 1996.

2. (a) Spagnolo, P.; Zanirato, P. J. Org. Chem. 1978, 43, 3539. (b) Spinelli, D.; Zanirato, P. J. Chem. Soc., Perkin Trans 2 1993, 1129. (c) Gronowitz, S.; Zanirato, P. J. Chem. Soc., Perkin Trans. 2, 1994, 1815.

3. (a) Davies, D.; Spagnolo, P.; Zanirato, P. J. Chem. Soc., Perkin Trans. 1 1995, 613. (b) Valenti, F.; Zanirato, P. J. Chem. Soc., Perkin Trans 2 1999, 623.

4. For recent reviews on this topic see: (a) Funicello, M.; Spagnolo, P.; Zanirato, P. Acta Chem. Scand. 1993, 47, 231. (b) Becher, J.; Dehaen, W. Acta Chem. Scand. 1993, 47, 244.

5. (a) Smith, P. A. S. Org. React. 1946, 3, 337. (b) Ah-Kow, G.; Paulmier, C.; Pastour, C. Bull. Soc. Chim. Fr. 1976, 151. (c) Binder, D.; Habison, G.; Noe, C.R. Synthesis 1977, 255. (d) Advanced Organic Chemistry, March, J., Ed., Wiley: New York, 4th Ed., 1992, p 547.

6. (a) Effenberger, F.; Gleiter, R. Chem. Ber. 1964, 97, 472. (b) Alder, R. W.; Chalkley, G. R.; Whiting, M. C., J. Chem. Soc. 1966, 52. (c) Norris, R. K. The Chemistry of Heterocyclic Compounds, Gronowitz, S., Ed.; 1990, 44, Part II, p 631. (d) Bailey, P. D.; Collier, I. D.; Morgan, K. M., Comprehensive Organic Functional Group Transformation, Katritzky, A. R.; Meth-Cohn, O.; Rees, C. W., Eds.; Pergamon: Cambridge, 1995, 5, 257. (f) Connell, R. D.; Comprehensive Organic Functional Group Transformation, Katritzky, A. R.; MethCohn, O.; Rees, C. W., Eds, Pergamon: Cambridge, 1995, Vol.5, 309.

7. Toselli, M.; Zanirato, P. J. Chem. Soc., Perkin Trans 1 1992, 1101.

8. Gribble, G. W.; Comprehensive Heterocyclic Chemistry II, Katritzky, A. R.; Rees, C. W.; Scriven E. F. V.; Storr, R. C., Eds, Pergamon: Oxford, 1996, Vol. 2, p 207.

9. (a) Damayanthi, Y.; Reddy, B. S. P.; Lown, J. W. J. Org. Chem. 1999, 64, 290. (b) Christiansen, L. E. E.; Selenophenes ref. 8, p 731. (c) Russel, R. K.; Press, J. B. ref. 8, p 679.

10. Weinstock, J. J. Org. Chem. 1961, 26, 3511.

11. Rault, S.; Cugnon de Sevricourt, M.; Dung, N-H.; Robba, M. J. Heterocyclic Chem. 1981, $18,739$.

12. Azides and Nitrenes; Scriven, E. F. V., Ed., Academic: Orlando, 1984, p 205.

13. Jones, G. B.; Chapman, B. J. ref. 8, 1. 